P83 A CASE OF HIV ASSOCIATED NEUROCOGNITIVE IMPAIRMENT (HAND) RESPONDING TO HAART SWITCH

Benjamin Goorney*, Suzannah Lant. Salford Royal Foundation Trust, Salford, Greater Manchester, UK

\subsection{6/sextrans-2015-052126.126}

Background/introduction We describe a case of a $34 \mathrm{yr}$ old Black African women fully suppressed on HAART for $9 \mathrm{yrs}$ presenting with recurrent episodes of HIV encephalopathy with abnormal MRI brain scan and detectable HIV in CSF. Following ARV switch her cognitive function and scans had improved and remains undetectable in CSF.

Aim(s)/objectives Started HAART in 2005 and remained asymptomatic and fully suppressed on (Kivexa/Atvr/rit) CD4 > 500 $\mathrm{mm}$. Presented initially in 2014 to Neurology with acute confusion, headaches and convulsions. CSF revealed pleocytosis with V/L 811 copies and neg for infective screen. MRI scan revealed diffuse non-specific signals consistent with HIV encephalopathy. On recovery she was monitored in clinic and remained virologically controlled but with residual neurocognitive impairment characterised by short term memory loss and difficulty concentrating. She then represented 9 months later with focal motor signs and confusion resolving within 48 hrs MRI scan no focal lesion. Rpt CSF revealed V/L of 960 copies.

Results In view of persistant CSF viraemia she was switched to higher CPE score (from 7 to 12) HAART regimen of Trizivir/ Maraviroc. Subsequently she fully recovered cognitive function and rpt CSF at 3/12 confirmed full suppressed VL with resolving brain scan.

Discussion/conclusion This case demonstrates that in well controlled pts on HAART who develop presumptive neuro-HIV and in absence of other potential causes, the value of CSF V/L in in constructing a HAART regimen with improved CSF penetration can result in significant improvement in both clinical and objective markers such as MRI scans.

\section{P84 NATIONAL HIV TESTING WEEK 3: FINDING THE HARD TO REACH AND BUILDING ON SUCCESS}

${ }^{1}$ Natasha Astill ${ }^{*},{ }^{2}$ Pat McCusker, ${ }^{3}$ Abigail Smith, ${ }^{4}$ Hannah Sowerbutts, ${ }^{2}$ Tom Doyle, ${ }^{3}$ Jeni Hirst, ${ }^{5}$ Jacqui Serbeh, 'Sarah Schoeman. 'Leeds Centre for Sexual Health, Leeds, UK; ${ }^{2}$ MESMAC, Leeds, UK; ${ }^{3}$ Skyline, Leeds, UK; ${ }^{4}$ Leeds City Council, Leeds, UK; ${ }^{5}$ Leeds Community Healthcare NHS Trust, Leeds, UK

\subsection{6/sextrans-2015-052126.127}

Background/introduction The third National HIV Testing Week (NHTW) was in November 2014.

Aims To build on our previous success of testing the most-atrisk populations (MARP), focusing on outreach.
Methods A third sector organisation, primarily targeting gay communities, provided club and bar outreach and offered pointof-care testing (POCT) on-site and at 2 saunas. A second third sector organisation, targeting African communities, offered POCT at 6 venues, including local markets, an asylum-seeker centre, pharmacies, health centres and an African football match. CASH services offered POCT at 3 clinics across the city. GUM and Leeds City Council staff volunteered to provide outreach and testing support for the 12 different testing sites across the city.

Results 167 people tested (126 in 2013, 94 in 2012). 71\% were from MARPs, unchanged from 2013. 1 female black African and 1 MSM tested HIV+ve, the first HIV diagnoses resulting directly from NHTW initiatives in our city. $74 \%$ of people who tested were sensitised through community outreach. Over $90 \%$ of people tested were given advice on PEP, repeat testing, STI screening and offered condoms.

Discussion Two undiagnosed HIV+ve people were identified as a result of NHTW efforts, and both are now in HIV care. A greater population, including those from MARPs tested. Of the MARPs, a higher percentage were testing for the first time. This may reflect decreased overall testing in MARPs, or that our NHTW 2014 campaign was more successful at reaching and testing people who are less likely to attend more traditional testing sites.

\section{P85 HIV TESTING IN A RURAL SCOTTISH HEALTHBOARD - HAS ANYTHING CHANGED?}

${ }^{1}$ Kirsty Abu-Rajab*, ${ }^{2}$ Mike Williamson. ${ }^{1}$ Central Sexual Health Department, Forth Valley, UK; ${ }^{2}$ Microbiology Department, NHS Forth Valley, UK

\subsection{6/sextrans-2015-052126.128}

Background/introduction Forth Valley NHS Health board is in central Scotland, covers a land area of $2633 \mathrm{~km}^{2}$ and looks after approximately 300,000 people. Education to healthcare professionals in different formats to try to increase HIV testing in those with relevant indicator conditions, in routine sexual health screens and in those from higher risk populations have been more frequent and visible in the last few years.

Aim(s)/objectives We were keen to see if this had resulted in a change in testing.

Methods A laboratory report showing the requesting location all HIV tests performed in 2012 and 2014 was produced. New HIV diagnoses attending the local HIV service and where they had been diagnosed was also recorded.

Results Overall a $19 \%$ increase in testing in 2014 compared to 2012.

\begin{tabular}{|c|c|c|c|c|c|c|c|c|c|c|c|c|}
\hline & \multirow{2}{*}{\multicolumn{2}{|c|}{ Total testing }} & \multirow{2}{*}{\multicolumn{2}{|c|}{$\begin{array}{l}1^{\text {st }} \text { ever } \\
\text { HIV test }\end{array}$}} & \multirow{2}{*}{\multicolumn{2}{|c|}{$\begin{array}{l}\text { If previously tested, } \\
\text { last test }>1 \text { yr ago }\end{array}$}} & \multirow{2}{*}{\multicolumn{2}{|c|}{ PEP aware }} & \multirow{2}{*}{\multicolumn{2}{|c|}{ Previously attended GUM }} & \multirow{2}{*}{\multicolumn{2}{|c|}{ Reactive test }} \\
\hline & & & & & & & & & & & & \\
\hline & 2013 & 2014 & 2013 & 2014 & 2013 & 2014 & 2013 & 2014 & 2013 & 2014 & 2013 & 2014 \\
\hline Black African & 27 & 49 & $19 \%$ & $29 \%$ & $55 \%$ & $43 \%$ & $19 \%$ & $20 \%$ & $36 \%(9 / 25)$ & $27 \%$ & 0 & 1 \\
\hline \multirow[t]{2}{*}{ MSM } & 61 & 70 & $39 \%$ & $47 \%$ & $39 \%$ & $35 \%$ & $32 \%$ & $50 \%$ & $46 \%$ & $47 \%$ & 0 & 1 \\
\hline & & & (21/54) & & & & & & $(26 / 57)$ & & & \\
\hline Total & 88 & 119 & $32 \%$ & $40 \%$ & $45 \%$ & $24 \%$ & $28 \%$ & $38 \%$ & $43 \%$ & $39 \%$ & 0 & $0.02 \%$ \\
\hline
\end{tabular}




\begin{tabular}{lll} 
Abstract P85 Table 1 & HIV testing in Rural Scotland & \\
\hline Testing Location & 2012 & 2014 \\
\hline Antenatal & 3583 & 3427 \\
Sexual health & 2668 & 3281 \\
General Practice & 425 & 890 \\
Ward mix & 288 & 209 \\
Gastoenterology outpatients & 305 & 357 \\
Renal & 206 & 261 \\
Occupational health & 230 & 244 \\
Termination of pregnancy & 253 & 247 \\
Prisons & 340 & 594 \\
General outpatients & 34 & 20 \\
Rheumatology & 10 & 103 \\
Haematology & 31 & 74 \\
Emergency department + Acute Assessment Unit & 51 & 64 \\
Addiction services & 64 & 267 \\
Paediatrics, ENT, Respiratory, Cardiology, Gynaecology, & 70 & 147 \\
ICU, Mental health, Maxillofacial, Neurology, Ophthalmology, & & \\
Orthopaedics, Dermatology, Needlestick source testing & & \\
Total & 8558 & 10185 \\
\hline
\end{tabular}

In 2012 there was one new HIV diagnosis, this was in the sexual health service. In 2014 there were four new diagnoses, two in sexual health and two in ENT.

Discussion/conclusion This work has been helpful to show where HIV testing is being performed. This work allows us to target specific departments and encourage relevant testing and optimise patient testing pathways. We plan to repeat this work as we are aware of current initiatives in several departments such as the acute admission unit. We will also compare our results with the four other health boards through the West of Scotland sexual health MCN. In future work we will also be able to look at 'Reasons for testing' as this will be clearly recorded using the new test order system.

\section{\begin{tabular}{|l|l}
\hline P86 AN AUDIT OF TIME TAKEN TO REACH UNDETECTABLE \\
\hline
\end{tabular} VIRAL LOAD IN THERAPY-NAÏVE HIV-POSITIVE PATIENTS INITIATING ART}

${ }^{1}$ Muhammad Ismail ${ }^{*},{ }^{2}$ Emmanuel Okpo, ${ }^{2}$ Steve Baguley, ${ }^{2}$ Ambreen Butt, ${ }^{2}$ Daniela Brawley, ${ }^{2}$ Ivan Tonna. 'University of Aberdeen, Aberdeen, UK; ${ }^{2}$ NHS Grampian, Aberdeen, UK

\subsection{6/sextrans-2015-052126.129}

Background The primary aim of antiretroviral therapy (ART) is to reduce morbidity and mortality due to chronic HIV infection. Central to ART is viral suppression, and this has been used as a proxy for disease burden. BHIVA guidelines recommend that patients achieve undetectable viral loads $(<50$ copies $/ \mathrm{mL})$ within 6 months of initiating ART.

Aim To assess the proportion of patients achieving undetectable viral loads within 6 and 12 months of initiating ART at a dualsite HIV service in Grampian.

Methods A retrospective case notes review was conducted of HIV-positive patients attending clinics between January 2013 and December 2013. Data was collected using a standardised proforma and imported into SPSS 23 for statistical analysis.

Results Twenty-four case notes were audited (GUM = 15, ID = 9). The median age of patients was 39.5 years. Median baseline viral load and CD4 count were 77,355 copies/mL and 382 respectively. Overall, $70.8 \%$ of patients achieved undetectable viral load within 6 months and 95.8\% achieved undetectable viral loads within 12 months (mean $=4.48$ months, 95\%
$\mathrm{CI}=3.50-5.70)$. A Kaplan-Meier survival analysis showed that patients with a baseline viral load of $<100,000$ copies $/ \mathrm{mL}$ achieved undetectable viral load sooner compared to those with $>100,000$ copies $/ \mathrm{mL}$ (3.43 months, 95\% CI $=2.34-3.66$ vs. 6.11 months, $95 \% \mathrm{CI}=4.28-7.94$; log-rank $\mathrm{p}=0.013$ ).

Conclusion This audit has identified potential barriers to viral suppression, such as late diagnosis and late commencement of ART. These areas must be addressed to ensure the target of $75 \%$ of patients with an undetectable viral load within 6 months of initiating ART can be achieved.

\section{P87 USE OF POCKET-SIZED HIV TESTING GUIDELINE CARDS TO INCREASE HIV TESTING IN MEDICAL INPATIENTS}

Chantal Oxenham*, Mithun Chakravorty, Ayman El-Nayal, Nadi Gupta. Rotherham NHS Foundation Trust, Rotherham, South Yorkshire, UK

\subsection{6/sextrans-2015-052126.130}

Background/introduction HIV is a chronic treatable condition with an excellent prognosis. There remains, however, a high morbidity and mortality due to late diagnosis, with approximately 1 in 4 HIV patients unaware of their condition. Healthcare professionals have previously seen many of these patients without the diagnosis being made. Rotherham's HIV prevalence is 1.05 per 1000 . Late diagnosis made in $56 \%$.

Aim(s)/objectives To increase HIV testing in general medical inpatients.

Methods We obtained a list of all medical inpatients in March 2014 who had been coded with a condition that should prompt HIV testing in accordance with BHIVA 2008 guidance.

We reviewed the number of HIV tests requested on medical inpatients during the 1-month period. In June 2014, we delivered a presentation at the Medical Grand Round and two subsequent teaching sessions for staff on HIV testing. We produced a pocket-sized card for staff to attach to the back of their ID badges listing the indications for testing. We compared the proportion of HIV tests performed before and after this intervention.

Results In March 2014, there were 69 patients with clinical indicators for HIV testing. Of those 32 were tested (46.4\%). In June 2014, following the intervention, there were 58 patients with clinical indicators and $40(69.0 \%)$ of those were tested.

Discussion/conclusion Following our educational intervention, almost $70 \%$ of patients were tested appropriately representing a $22.6 \%$ increase from baseline. We plan to re-measure this at a later date to assess whether this increase in uptake of testing has been sustained.

\section{P88 ROUTINE HIV TESTING IN ACUTE GENERAL MEDICINE USING A NON-PHYSICIAN IMPLEMENTED MODEL}

${ }^{1}$ Amina Buba*, ${ }^{2}$ Martin Fisher, ${ }^{2}$ Colin Bentley, ${ }^{2}$ Jonathan Roberts, ${ }^{2}$ Elaney Youssef, ${ }^{2}$ Mohammed Hassan Ibrahim, ${ }^{1}$ Michael Brown, ${ }^{2}$ Daniel Richardson. 'London School of Hygiene and Tropical Medicine, London, UK; ${ }^{2}$ Brighton and Sussex University Hospitals, East Sussex, UK

\subsection{6/sextrans-2015-052126.131}

Background/introduction UK national guidelines recommend routine HIV testing in general medical admissions and primary care in areas where the HIV prevalence exceeds $2 / 1000$ in the local population. The guidelines recommend further operational research to assess the feasibility and efficacy of different 